\title{
Sur quelques transformations d'une série de puissances.
}

\author{
(Par Niels Nielsex, à Copenhague.)
}

\section{§ 1. Formulegs ańmbrates.}

Supposons pour un instant que la variable $x$ soit réelle et que $0 \leqq x \leqq 1$, puis mettons dans cette intégrale indéfinie

$$
J=\int \frac{f(x)}{x \sqrt{1-x^{2}}} d x
$$

$x=\sin \varphi$, où $\varphi$ désigne un angle réel tel que $0 \leqq \varphi \leqq \frac{\pi}{2}$, une intégration par parties donnera immédiatement

$$
J=f(\sin \varphi) \cdot \log \operatorname{tg} \frac{1}{2} \varphi-\int f^{(1)}(\sin \varphi) \cdot \cos \varphi \cdot \log \operatorname{tg} \frac{1}{2} \varphi d \varphi,
$$

de sorte que nous aurons, à l'aide de la nouvelle transformation $\operatorname{tg} \frac{1}{2} q=z$, pour notre intégrale cette autre expression

$$
J=f\left(\frac{2 z}{1+z^{3}}\right) \log z-2 \cdot \int f^{(1)}\left(\frac{2 z}{1+z^{2}}\right) \cdot \frac{1-z^{2}}{\left(1+z^{2}\right)^{2}} \cdot \log z d z .
$$

Supposons maintenant que la fonction $f(x)$ soit holomorphe aux environs du point $x=0$ et que la série de puissances correspondante ait son rayon de convergence égal à $r$, nous aurons cette autre série de puissances

$$
\frac{f(x)}{\sqrt{1-x^{2}}}=a_{0}+a_{1} x+a_{2} x^{2}+a_{3} x^{3}+\cdots,|x|<\rho,
$$

où $\rho$ désigne le plus petit des deux nombres positifs 1 et $r$, ce qui donnera Annali di Matematica, Serie III, tomo X. 
immédiatement

$$
J-a_{0} \log x=\frac{a_{i}}{1} \cdot x+\frac{a_{2}}{2} \cdot x^{2}+\frac{a_{3}}{3} \cdot x^{3}+\cdots,|x|<\rho .
$$

Cela posé, il est évident que la fonction $f\left(\frac{2 z}{1+z^{2}}\right)$ est holomorphe aussi aux environs du point $z=0$, de sorte que nous aurons cette nouvelle série de puissances

$$
f\left(\frac{2 z}{1+z^{2}}\right)=a_{0}+b_{1} z+b_{2} z^{2}+b_{3} z^{3}+\cdots,|z|<P
$$

ce qui donnera

$$
f^{(1)}\left(\frac{2 z}{1+z^{2}}\right) \cdot \frac{1-z^{2}}{\left(1+z^{2}\right)^{2}}=b_{1}+2 b_{2} z+3 b_{3} z^{2}+\cdots,|z|<P .
$$

Quant au rayon de convergence $P$, nous aurons à donner plus tard pour cette quantité des limites supérieures et inférieures.

Appliquons maintenant ici cette formule intégrale

$$
\int x^{n} \log x d x=\frac{x^{n+1}}{n+1} \cdot \log x-\frac{x^{n+1}}{(n+1)^{2}},
$$

nous aurons, en vertu de $(\gamma)$, cette nouvelle formule

$$
J-a_{0} \log z=\frac{b_{1}}{1} \cdot z+\frac{b_{2}}{2} \cdot z^{2}+\frac{b_{1}}{3} \cdot z^{3}+\cdots,|z|<P .
$$

Or, remarquons que les deux variables $x$ et $z$ sont liées par cette identité

$$
x=\frac{2 z}{1+z^{2}}
$$

Jes formules (2) et ( 2 bis) donnent, en vertu d'un théorème fondamental de la théorie des fonctions analytiques, une identité de cette forme

$$
a_{0} \log \left(\frac{2}{1+z^{2}}\right)+\sum_{s=1}^{s=\infty} \frac{a_{s}}{s} \cdot\left(\frac{2 z}{1+z^{2}}\right)^{s}=\sum_{s=1}^{s=\infty} \frac{b_{s}}{s} \cdot \hat{z}^{s}+K,
$$

où $K$ désigne une constante arbitraire. Pour déterminer la valeur de cette constante mettons dans $(\delta) z=0$, ce qui est permis, nous aurons $K=\log 2$. Posons ensuite $x$ au lieu de $z$, nous avons démontré ce théorème général:

I. Définissons, à l'aide de (1) et (1 bis), les deux groupes de coefficients $\alpha_{s}$ et $b_{s}$, nous aurons cette identité:

$$
\sum_{s=1}^{s=\infty} \frac{a_{s}}{s} \cdot\left(\frac{2 x}{1+x^{2}}\right)^{s}-a_{0} \log \left(1+x^{2}\right)=\sum_{s=1}^{s=\infty} \frac{b_{s}}{s} \cdot x^{s},
$$


formule qui nous donnera le prolongement analytique de la série de puissunces figurant au second membre et étant convergente pour des valeurs suffisamment petites de $|x|$.

Considérons encore cette autre intégrale indéfinie

$$
J_{1}=\int \frac{g(x)}{x\left(1+x^{2}\right)} d x
$$

la transformation $x=\operatorname{tg} \varphi$ nous conduira, à l'aide d'une intégration par parties, à cette nouvelle expression

$$
J_{1}=g(\operatorname{tg} \varphi) \cdot \log \sin \varphi-\int g^{(1)}(\operatorname{tg} \varphi) \cdot \frac{\log \sin \varphi}{\cos ^{3} \varphi} d \varphi
$$

d'où, en posant $\sin \varphi=z$ :

$$
J_{1}=g\left(\frac{z}{\sqrt{1-z^{2}}}\right) \cdot \log z-\int g^{(1)}\left(\frac{z}{\sqrt{1-z^{2}}}\right) \cdot \frac{\log z}{\left(1-z^{2}\right)^{\frac{3}{2}}} d z
$$

Supposons ensuite que la fonction $g(x)$ soit holomorphe aux environs du point $x=0$ et que la série de puissances correspondante ait son rayon de convergence égal à $r$, nous aurons ici ces deux autres séries de puissances:

$$
\begin{aligned}
\frac{g(x)}{1+x^{2}} & =c_{0}+c_{1} x+c_{2} x^{2}+c_{3} x^{3}+\cdots,|x|<\rho, \\
g\left(\frac{z}{\sqrt{1-z^{2}}}\right) & =c_{0}+d_{1} z+d_{2} z^{2}+d_{3} z^{3}+\cdots,|z|<P_{3},
\end{aligned}
$$

où $\rho$ désigne le plus petit des nombres positifs 1 et $r$, tandis que nous avons à donner bientôt des limites supérieures et inférieures de $P_{1}$.

Cela posé, nous aurons sans peine cet autre théorème général :

II. Définissons, à l'aide de (4) et (4 bis), les deux groupes de coefficients $c_{s}$ et $d_{s}$, nous aurons cette identité:

$$
\sum_{s=1}^{s=\infty} \frac{c_{s}}{s} \cdot\left(\frac{x}{\sqrt{1-x^{2}}}\right)^{s}-c_{0} \log \left(\sqrt{1-x^{2}}\right)=\sum_{s=1}^{s=\infty} \frac{d_{s}}{s} \cdot x^{s},
$$

formule qui nous donnera le prolongement analytique de la série de puissances figurant au second membre et étant convergente pour des valeurs suffisamment petites de $|x|$.

Supposons données les deux séries de puissances figurant aux seconds membres des formules (3) et (5), ou, ce qui vaut autant, les coefficients $b$, 
et $d_{s}$, la détermination des $a_{s}$ et des $b_{s}$ deviendra très compliquée, de sorte qu'il est très remarquable que la forme singulière que nous avons donnée aux formules susdites nous conduira immédiatement au but dans un nombre des cas intéressants. Or, avant d'étudier de tels cas particuliers, nous avonṣ à déterminer les champs de convergence des séries de Burmans qui figurent aux premiers membres de $(3)$ et $(5)$ et à donner des limites pour les deux rayons de convergence $P$ et $P_{1}$, si nous supposons données les fonctions $f(x)$ et $g(x)$.

\section{$\S$ 2. SUR LES CHAMPS DE CONVERGENCE.}

Quant au champ de convergence de la série de Burmann figurant au premier membre de la formule (3), remarquons que la fonction

$$
F(z)=f\left(\frac{2 z}{1+z^{2}}\right)
$$

est holomorphe dans tous les points du plan des $z$ pour lesquels

$$
\left|\frac{2 z}{1+z^{2}}\right|<r
$$

posons maintenant $z=\xi+i \eta$, où $\xi$ et $\eta$ désignent les coordonnées rectangles du point $z$, l'inégalité $(\alpha)$ nous conduira à cette autre

$$
\left(\xi^{2}+n^{2}\right)^{2}-2\left(\frac{2}{r^{2}}-1\right) \xi^{2}-2\left(\frac{2}{r^{2}}+1\right) \eta^{2}+1>0 .
$$

Cela posé, considérons cette courbe $C(\boldsymbol{r})$ :

$$
\left(x^{2}+y^{2}\right)^{2}-2 a x^{2}-2(a+2) y^{2}+1=0, \quad a=\frac{2}{r^{2}}-1
$$

on bien en coordonnées polaires

$$
\rho^{4}-2\left(a+2 \sin ^{2} \theta\right) \rho^{2}+1=0,
$$

nous verrons que $C(r)$ est une courbe fermée qui a des points doubles dans les points circulaires à l'infini.

Or, la condition $(\alpha)$ montre clairement que $F(z)$ est certainement holomorphe dans tous les points du plan des $z$ que l'on peut obtenir en prenant comme point de départ $z=0$ et en suivant un chemin fermé qui a un nombre 
pair de points d'intersection avec $C(\boldsymbol{r})$. Nous désignons par $\mathfrak{B}(\boldsymbol{r})$ l'ensemble de tous ces points; c'est-à-dire que $F(z)$ est certainement holomorphe dans la partie $\mathfrak{B}(r)$ du plan des $z$. Inversement, il est évident que $F(z)$ a toujours un point singulier au moins situé sur la courbe $C(r)$, ce qui montrera que les limites supérieures et inférieures de $P$ seront précisément le plus grand et le plus petit rayon vecteur qui unit l'origine avec un point de $C(\boldsymbol{r})$.

Considérons maintenant l'équation ( 6 bis), il est évident que les valeurs maximums et minimums de $\rho$ sont celles qui correspondent aux points d'intersection de $C(r)$ avec les axes de coordonnées. De plus, je dis que ces valeurs nous donnent aussi le maximum et le minimum absolus de $\rho$.

En effet, cherchons $\sin \theta$ de $(6 \mathrm{bis})$, la condition nécessaire èt suffisante pour que l'angle $\theta$ soit réel deviendra

$$
0 \leqq \rho^{4}-2\left(\frac{2}{r^{2}}-1\right) \rho^{2}+1 \leqq 4 \rho^{2}
$$

ce qui donnera pour $P$ ces deux limites

$$
\sqrt{\frac{1}{r^{2}}+1}+\frac{1}{r} \leqq P \leqq \sqrt{\frac{1}{r^{2}}+1}-\frac{1}{r}
$$

limites qui coïncident pour $r=\infty$, e'est-à-dire $a=-1$, et dans ce cas seulement.

Cela posé, nous avons démontré cette proposition générale:

L'identité (3) nous donne le prolongement analytique à tout le domaine $\mathfrak{B}(\rho)$ ( $\rho$ étant le rayon de convergence défini dans (1)) de la série de puissances figurant au second membre de la formule susdite et dont le rayon de convergence doit satisfaire aux conditions (7).

Quant au théorème II, considérons la fonction

$$
G(z)=g\left(\frac{z}{\sqrt{1-z^{2}}}\right)
$$

nous trouvons ici cette courbe $C_{1}(r)$ :

$$
(a-1)\left(x^{2}+y^{2}\right)^{2}-2 a\left(x^{2}-y^{2}\right)+a=0, \quad a=r^{4},
$$

ou bien, en coordonnées polaires

$$
(a-1) p^{4}-2 a \rho^{2} \cos 2 \theta+a=0 .
$$

Supposons $r \gtrless 1$, la courbe $C_{1}(r)$ est fermée et a des points doubles dans 
les points circulaires à l'infini. Dans le cas particulier $r=1$, au contraire, notre courbe deviendra une hyperbole équilatérale avec son axe égal à $\sqrt{\frac{1}{2}}$.

Le même procédé que nous venons d'appliquer nous conduira jei à ces valeurs limites:

$$
\begin{gathered}
\sqrt{\left|\frac{r^{4}+r^{2}}{r^{4}-1}\right|} \geqq P_{1} \geq \sqrt{\frac{r^{4}-r^{2}}{r^{4}-1}}, \quad r \geq 1, \\
\rho \geqq \sqrt{\frac{1}{2}}, \quad r=1 .
\end{gathered}
$$

Désignons ensuite par $\mathfrak{P}_{(}(r)$ l'ensemble des points du plan des $z$ que l'on peut obtenir en partant de $z=0$ et en suivant un chemin fermé qui a un nombre pair de points d'intersection avec $C_{1}\left(v^{\prime}\right)$, nous avons démontré cette autre proposition:

La formule (5) nous donne le prolongement analytique $\grave{a}$ tout le domaine $\Re_{1}(\rho)$ ( $\rho$ étant le rayon de convergence défini dans $(4)$ ) de la série de puissances figurant au second membre de lá formule susdite et dont le rayon de convergence doit satisfaire aux conditions (9) et (9 bis).

\section{§. Géneralisation d'une formule de M. Kaptexn.}

Comme premier exemple de la transformation indiquée dans le théorème I posons $f(x)=1$, ce qui donnera

$$
\rho=1, \quad a_{0}=1, \quad a_{2 s}=\frac{1.3 .5 \ldots(2 s-1)}{2.4 .6 \ldots(2 s)}, \quad a_{2 s+1}=0, \quad b_{s}=0,
$$

de sorte que la formule (3) donnera ici ce développement particulier

$$
\log \left(1+x^{2}\right)=\sum_{s=1}^{s=\infty} \frac{1.3 .5 \ldots(2 s-1)}{2.4 .6 \ldots(2 s)} \cdot \frac{1}{2 s} \cdot\left(\frac{2 x}{1+x^{2}}\right)^{2 s}
$$

valable dans le domaine $\mathfrak{P}(1)$. Posons particulièrement $x=1$, ce qui est permis, nous aurons cette formule numérique

$$
\log 2=\sum_{s=1}^{s=\infty} \frac{1.3 .5 \ldots(2 s-1)}{2.4 .6 \ldots(2 s)} \cdot \frac{1}{2 s} .
$$


L'hypothèse correspondante $g(x)=1$ nous conduira, en vertu de (5), à une identité formelle.

Pour obtenir des applications plus générales de nos deux théorèmes généraux posons

puis mettons

$$
\left.\begin{array}{l}
\frac{(\arcsin x)^{n}}{n !}=\sum_{s=0}^{s=\infty} A_{n}^{n+2 s} \cdot x^{n+2 s},|x| \leqq 1, \\
\frac{(\operatorname{arctg} x)^{n}}{n !}=\sum_{s=0}^{s=\infty} B_{n}^{n+2 s} x^{n+2 s},|x| \leqq 1,
\end{array}\right\}
$$

nous aurons évidemment

$$
f(x)=\frac{(\arcsin x)^{n}}{n !}
$$

$$
f\left(\frac{2 z}{1+z^{2}}\right)=\frac{2^{n}}{n !} \cdot(\operatorname{arctg} z)^{n}
$$

de sorte que les coefficients correspondants $a_{s}$ et $b_{s}$ se déterminent comme suit :

$$
\left.\begin{array}{l}
a_{s}=0, \quad s<n ; \quad a_{n+2 s}=(n+2 s+1) \cdot A_{n+1}^{n+2 s+1} ; \quad a_{n+2 s+1}=0 \\
b_{s}=0, \quad s<n ; \quad b_{n+2 s}=2^{n} \cdot B_{n}^{n+2 s} ; \quad b_{n+2 s+1}=0 .
\end{array}\right\}
$$

En second lieu mettons

nous aurons

$$
g(x)=\frac{(\operatorname{arctg} x)^{n}}{n !}
$$

$$
g\left(\frac{z}{\sqrt{1-z^{2}}}\right)=\frac{(\arcsin z)^{n}}{n !}
$$

ce qui donnera pour les coefficients $c_{s}$ et $d_{s}$ ces systèmes de valeurs:

$$
\left.\begin{array}{l}
c_{s}=0, \quad s<n ; \quad c_{n+2 s}=(n+2 s+1) . B_{n+1}^{n+2 s+1} ; \quad c_{n+2 s+1}=0 \\
d_{s}=0, \quad s<n ; \quad d_{n+2 s}=A_{n}^{n+2 s} ; \quad d_{n+2 s+1}=0 .
\end{array}\right\}
$$

Cela posé, une application directe des deux théorèmes généraux donnera cette proposition intéressante:

Définissons à l'aide des formules (12) et (12 bis) les coefficients $A$ et $B$, nous aurons ces deux formules singulieres:

$$
\begin{aligned}
& \sum_{s=0}^{s=\infty} \frac{n+2 s+1}{n+2 s} \cdot A_{n+1}^{n+2 s+1} \cdot\left(\frac{2 x}{1+x^{2}}\right)^{n+2 s}=2^{n} \cdot \sum_{s=0}^{s=\infty} \frac{B_{n}^{n+2 s}}{n+2 s} \cdot x^{n+2 s}, \\
& \sum_{s=0}^{s=\infty} \frac{n+2 s+1}{n+2 s} \cdot B_{n+1}^{n+2 s+1} \cdot\left(\frac{x}{1-x^{2}}\right)^{n+2 s}=\sum_{s=0}^{s=\infty} \frac{A_{n}^{n+2 s}}{n+2 s} \cdot x^{n+2 s},
\end{aligned}
$$


où $P=P_{1}=1$, et où les séries de Burmann sont convergentes dans les domaines $\mathfrak{B}(1)$ et $\mathfrak{B}_{1}(1)$ respectivement.

Posons particulièrement dans (13) $n=1$, puis mettons

$$
\psi(x)=\frac{x}{1^{2}}-\frac{x^{3}}{3^{2}}+\frac{x^{5}}{5^{2}}-\frac{x^{7}}{7^{2}}+\cdots
$$

nous aurons cette formule particulière

$$
2 \cdot \psi(x)=\frac{2}{1+x^{2}}+\sum_{s=1}^{s=\infty} \frac{2 \cdot 4 \cdot 6 \ldots(2 s)}{3 \cdot 5 \cdot 7 \ldots(2 s+1)} \cdot \frac{1}{2 s+1} \cdot\left(\frac{2 x}{1+x^{2}}\right)^{2 s+1},
$$

d'où en posant $x=1$, cette relation numérique

$$
2 \cdot \sum_{s=0}^{s=\infty} \frac{(-1)^{s}}{(2 s+1)^{2}}=1+\sum_{s=1}^{s=\infty} \frac{2.4 .6 \ldots(2 s)}{3.5 .7 \ldots(2 s+1)} \cdot \frac{1}{2 s+1}
$$

que j'ai démontrée directement $\left(^{*}\right)$ autrefois, tandis que la formule plus générale (14) est due à M. W. Kaptexn (**).

\section{§ 4. Généralisation d'Une formdie d'Abel.}

On voit que la fonction $\psi(x)$ figurant dans (14) est intimément liée avec cette fonction célèbre

$$
L_{2}(x)=\frac{x}{1^{2}}+\frac{x^{2}}{2^{2}}+\frac{x^{3}}{3^{2}}+\frac{x^{4}}{4^{2}}+\cdots,|x|<1,
$$

pour laquelle Legendre (***), ABEu (****) et Schaefrers (*****) ont démontré une suite de propriétés remarquables et de laquelle j'ai ètudié récemment (******) des généralisations très étendues. Or, il est intéressant, ce me semble, que notre théorème II nous permet de généraliser beaucoup une formule d'ABEL concernent la fonction $L_{2}(x)$.

(*) Nyt Tidsskrift for Matematik, t. 5 B, p. 24; 1894.

(*) Nieuw Archief, (2) t. 3, p. 225-220; 1897.

(**) Exercices de calcul différentiel et intégral, t. II, p. 244.

(****) Eivvres complètes, t. II, p. 189-193.

(******) Journal de Crelle, t. XXX.

$(* * * * * *)$ Ce Journal, $3^{\ominus}$ série, t. IX, p. 219-236; 1903. 
En effet, désignons par $\omega_{n}^{p}$ la somme de tous les $\left(\begin{array}{l}n \\ p\end{array}\right)$ produits à $p$ facteurs différents choisis parmi ces $n$ nombres positifs

d'où particulièrement

$$
\frac{1}{1}, \frac{1}{2}, \frac{1}{3}, \ldots, \frac{1}{n},
$$

$$
\omega_{n}^{1}=\frac{1}{1}+\frac{1}{2}+\cdots+\frac{1}{n}, \quad \omega_{n}^{n}=\frac{1}{n !},
$$

tandis que nous posons en outre

$$
\omega_{n}^{0}=1 \text {. }
$$

Cela posé, il est bien connu $\left(^{*}\right)$ que nous avons cette série de puissances

$$
\frac{(-1)^{n}}{n !} \cdot(\log (1-x))^{n}=\sum_{s=0}^{s=\infty} \frac{\omega_{n+s-1}^{n-1}}{n+s} \cdot x^{n+s}, \quad|x|<1,
$$

et où $n$ désigne un positif entier.

Posons encore

$$
L_{1, n}(x)=\sum_{s=0}^{s=\infty} \frac{\omega_{n+s-1}^{n-1}}{(n+s)^{2}} \cdot x^{n+s}, \quad|x|<1,
$$

nous aurons évidemment

$L_{1, n}(x)=\frac{(-1)^{n}}{n !} \cdot \int_{0}^{1} \frac{(\log (1-t x))^{n}}{t} d t=\frac{(-1)^{n}}{n !} \cdot \int_{0}^{x} \frac{(\log (1-x))^{n}}{x} d x,(15 \mathrm{bis})$ où il faut admettre $|x|<1$; en particulier nous aurons de plus

$$
L_{i, 1}(x)=L_{2}(x) \text {. }
$$

Ponsons maintenant dans le théorème II

$$
g(x)=\frac{1}{n !} \cdot\left(\log \left(1+x^{2}\right)\right)^{n}
$$

nous aurons évidemment

$$
g\left(\frac{z}{\sqrt{1-z^{2}}}\right)=\frac{(-1)^{n}}{n !} \cdot\left(\log \left(1-z^{2}\right)\right)^{n}
$$

tandis que la formule obtenue de $(\alpha)$ en $\mathrm{y}$ posant $-x^{2}$ au lieu de $x$

(*) Voir, par exemple, SchlömIrch : Compendium, t. II, p. 13. 
donnera

$$
J_{1}=\sum_{s=0}^{s=\infty} \frac{(-1)^{s} \omega_{n+s}^{n}}{n+s} \cdot x^{2 n+2 s}, \quad|x|<1 .
$$

Or, appliquons ces identités évidentes

$$
\begin{aligned}
& \omega_{n+s}^{n}=\frac{1}{n+s} \cdot \omega_{n+s-1}^{n-1}+\omega_{n+s-1}^{n}, s \geqq 1, \\
& \omega_{n}^{n}=\frac{1}{n} \cdot \omega_{n-1}^{n-1},
\end{aligned}
$$

nous aurons, en vertu de $(\alpha)$ et $(\beta)$ :

$$
J_{1}=(-1)^{n} L_{1, n}\left(-x^{2}\right)-\frac{1}{(n+1) !} \cdot\left(\log \left(1+x^{2}\right)\right)^{n+1},
$$

tandis que ( 4 bis) donnera, en vertu de $(\alpha)$, pour notre intégrale $J_{1}$ cette autre expression

$$
J_{i}=L_{1, n}\left(x^{2}\right)
$$

de sorte que la formule générale (5) nous conduira à cette identité intéressante

$$
L_{1, n}\left(\frac{x^{2}}{x^{2}-1}\right)-(-1)^{n} L_{1, n}\left(x^{2}\right)=-\frac{1}{(n+1) !} \cdot\left(\log \left(1-x^{2}\right)\right)^{n+1},
$$

d'où, en posant $-x$ au lieu de $x^{2}$ :

$$
L_{i, n}\left(\frac{x}{1+x}\right)-(-1)^{n} L_{1, n}(-x)=-\frac{1}{(n+1) !} \cdot(\log (1+x))^{n+1}
$$

On voit que la formule (17) prolonge la fonction $L_{i, n}\left(x^{2}\right)$ dans tout le domaine $\Re_{1}(1)$, tandis que (18) prolonge $L_{\imath, n}(-x)$ dans le demi-plan déterminé par la condition $\Re(x)>-\frac{1}{2}$. Or, comme je viens de le démontrer $\left(^{*}\right)$, la fonction $L_{1, n}(x)$ n'a dans toute l'éntendue du plan des $x$ que ces deux points critiques $x=1$ et $x=\infty$.

Posons dans (18) particulièrement $n=1$, nous retrouvons, en vertu de (16), une formule qui est due à Aber (**).

Copenhague, le 18 novembre 1903.

(*) Loc. cit., p. 221.

(**) Loc. cit., p. 191. 